\title{
VIII.
}

\section{Das vierte Jahrbuch der Schopenhauer-Gesellschaft.}

\section{Von \\ Maria Groener (Kastelruth, Tirol).}

Schopenhauer sagt von der philosophia prima gleich zu Anfang seiner Vorlesungen (Piper IX S. 29), daß sie eine notwendige Vorschule für die Philosophie oder Metaphysik sei. Er nennt sie das Medium, durch welches die Philosophie uns gegeben ist und verlangt daher ihre gründliche Kenntnis.

So erfreulich es nun ist, dảß das Jahrbuch der SchopenhauerGesellschaft von Jahr zu Jahr ernster und sachlicher wird, so bedauerlich finde ich seine Einseitigkeit in der bloßen Beschäftigung mit Schopenhauers Metaphysik der Natur, des Schönen und der Sitten, indes Schopenhauers philosophia prima links liegen gelassen wird. Der Schlußaufsatz des neuen Jahrbuchs von Arnold Samuelson stellt wieder einmal klar die Forderung auf: „Zuerst den Satz vom Grunde lesen!"

Wie wenig Menschen aber gibt es, die, wenn sie ehrlich sein wollen, sagen können, sie hätten den Satz vom Grunde wirklich und durchaus verstanden! -

Hier wäre eine große, schöne Aufgabe für die Gelehrten in der Gesellschaft: Schreibt für die Laien Einführungen, Erläuterungen zur philosophia prima Schopenhauers! - Statt dessen beschäftigen sich, abgesehen von den philologischen oder ganz persönlichen Aufsätzen, alle eigentlich philosophischen Arbeiten des Jahrbuchs bloß mit jenem zweiten Teil der Schopenhauerschen Philosophie, den jeder ernstlich Strebende ohne viel Kommentar zu verstehen vermag, sobald er nur die philosophia prima richtig erfaßt hat.

Wollten die philosophischen Aufsätze wenigstens zur Vertiefung des Verständnisses der Schopenhauerschen Philosophie beitragen, sie wären immerhin zu begrüßen. Leider aber haben es mehrere unter ihnen darauf abgesehen, Schopenhauern am Zeuge zu flicken. Der Grund dieser bedauerlichen Tatsache liegt darin, daß ihre Verfasser 
dir philosophia prima sich nicht assimiliert haben und daher Widerspriiche und Fehler zu finden glauben, wo in Wahrheit keine sind, oder da Gegensïtze aufzuzcigen versuchen, wo in Wahrheit höchste finigung herrscht.

Wie in den vorhergehenden Jahrbuichern, so ist auch in diesem (in großer Teil der Aufsät\%e Untersuchungen über Schopenhauers Metaphysik der Sitten gewidmet.

So beschüftigt sich Deussens Aufsatz (Wiedergabe des in Miunchen gehaltenen Vortrags) mit Schopenhauers Verhältnis zur Religion, Georg Grimm bespricht Thema und Basis der Lehre Buddhas, Karl Nebel schreibt uiber Schopenhauer und die brahmanische Religion.

Mit bewundernswertem Freimut weist Deussen schon im 'Vorwort zum Jahrbuch darauf hin, daß Grimm ihn hart tadelt, weil er den Buddhaismus als fiur unsere Zeit nicht mehr passend erkläre; im übrigen aber ist Deussen mit Grimm wie auch wohl mit Nebel ganz einverstanden, denn ein Tiefstes eint diese drei Männer: sie haben eine gemeinsam gegen Schopenhauer: gerichtete Spitze: Sie lassen die Wiedergeburt nicht mehr mit Schopenhauer als plötzliches Wunder gelten, sondern sie erklären die Willenswendung für. ,einen durch das ganze Leben sich hinziehenden Läuterungsprozeß" (S. 14, $66,68 ; 178)$.

Eine scheinbar kleine Abweichung von . Schopenhauer; von welcher Tragweite aber ist sie! Nach Schopenhauer ist „das Mysterio von der theologischen Gnade oder philosophischen Freiheit d.e.r Weltknoten".

Diesen unlösbaren Weltknoten glauben jene :drei Männer lösen zu können: aus der theologischen Gnade machen sie eine menschliche Lüuterung, Besserung, Selbsterlösung; aus der philosophischen Freiheit machen sie eine empirische.- Die Folge davon ist; daß wir kalt bleiben beim Lesen jener Aufsätze. Ein eigentümliches Gefühl, wie wir es in den Kindertagen bei moralisierenden Erzählungen hatten, beschleicht uns: wir fühlen, hier wird Moral gepredigt, aber nicht Hunger nach Wahrheit, nach Frieden gestillt: hier wird nichts Beglückendes, Befreiendes dargeboten.

Und die Ursache? Die philosophia prima ist nicht aswimiliert. Schopenhauers Unterscheidung. zwischen Wollen . und. Handeln, und seine-Lehre vom Zusammenbestehen der transzendentalen. Freiheit 
mit der empirischen Notwendigkei: ist darum nicht klar erfaßt, weil der Begriff der Notwendigkeit selbst in Schopenhauers Aposteln nicht wahre Gestalt in Schopenhauerschem Geiste gevonnen hat. Deshalb werden Schopenhauers wichtigste Deutungen christlicher Dogmen: die Deutung der Erbsünde, der Gnade, sowie der Trinität. als nebensächlich behandelt. Aus Schopenhauers Durchdringung der Dogmen wird eine bloße Berïhrung, aus seiner Röntgendurchstrahlung bloße Beleuchtung, aus seiner universellen Auslegung eine bloße Morallehre, und auf solche Weise wird der lebendige Strom Schopenhauerscher Gedanken künstlich in ein totes Geleise gelenkt.

Aber jener Irrium wird nicht allein für die Einschätzung Schopenhauers vẻrhängnisvoll; er führt auch zu einer Scheuklappenbeurteilung der indischen Gedankenwelt und zu einer Verstümmelung christlicher Lehrbegriffe.

In sehr vielen Stellen der Upanishads wird gelehrt, daß die höchste Erkemntnis nicht die des Geistes (Traum), sondern die des Tiefschlafs ist. (Siehe Deussen, Upanishads, z. B. 196, 411, 470). Den gleichen Gegensatz zwischen intuitivem und abstraktem Erkennen bringt Meister Eckhart, wennn er, wunderbar tiefsinnig und einleuchtend zugleich, von „überformet Wissen“ spricht (Pfeiffer 1857, S. 15/5). Denselben Gedanken finden wir auch schon in Jesu Gespräch mit Nicodemus: Spiritus ubi vult spirat: et vocem eius audis, sed nescis unde veniat, aut quo vadat: sic est omnis, qui natus est ex spiritu (Jo. 3, 8). Das aber ist noch nicht das Höchste, die Geburt vom Geiste; die wahre Wiedergeburt oder die Geburt von oben ist die ạus Geist und Wasser (Jo. 3, 5), eine. Geburt, die der Mensch als solcher nicht in sich vollziehen kann, denn wie Schopenhauer sagt, können wir nie deu Willen selbst ändern, sondern nur veranlassen, daß er das, was er so unablässig und unveränderlich sucht, auf einem andern Wege suche als bisher (W. 347).

Ganz klar liegt auch in ungezählten Stellen der Reden Gotama - Buddhas der Gedanke von der Unveränderlichkeit des Charakters; nur wird er von den einseitig gerichteten Interpreten zugunsten jener Stellen übersehen, die von einer moralischen Besserung reden. So z. B. Im Elefantengleichnis (Neumann, S. 274). Da heißt es, daß es dem Königssohn unmöglich ist, zur Erlösung zu kommen. Dies „,unmöglich", das so oft wiederkehrt, ist ebenso wichtig wie sein Gegenteil. Wer schon als Mönch, zum Mönch geboren ist, der wird 
dahin kommien, Mönch zu werden, wer aber, Mönch zu werden, nicht wollen kann, fïr den ist's unmöglich. Oder im Felsengleichnis, clas Grinm S. $67 \mathrm{f}$. eigens anführt, um den vermeintlichen Gegensatz zu Schopenhauer darzulegen: der Eine steht oben und sieht die Aussicht ins gelobte Land; der Andre, unten, sieht sie nicht. Führt ihn dann der Freund hinauf, so sieht er die Herrlichkeiten auch.

Also, so folgern die modernen Interpreten, lehrte schon Buddha, daß moralische Besserung möglich sei. Die Lehre von der Unveränderlichkeit des Charakters sei falsch. Der wahre Kenner Schopenhauers aber folgert: Wenn der unten dem Freund nicht Glauben zu schenken vermag, daß man oben Beseligendes erlebt und weun er selbst nicht hinauf will, so bringt man ihn nicht hinauf. Und zerrte man ihn nach oben, so würde er oben die Achseln zucken und den Ausblick nicht lohnend finden. Da liegt die Unveränderlichkeit des ('harakters.

Diese vereinzelten Beispiele können natürlich noch nicht ïberzeugend wirken. Sie sollen nur anregen. Sie sollen darauf hinweisen, daß wir mit solch einseitiger Deutung das Lebenswerk Schopenhauers geradezu im Kern vernichten. Er, der erste und einzige, der die Antinomie der Freiheit des Willens und der Unfreiheit des Handelns glïcklich gelöst und das, was die Inder und die christlichen Mystiker in Form von gefühlter Wahrheit besaßen, zu einer beweisbaren machte, wird von seinen Aposteln veroberflächlicht, verstümmelt, zerteilt und willkürlich beschnitten. Seine wunderbare Lösung wird nicht verstanden, ja, abfällig beurteilt.

Dies mußte einmal klar gesagt werden.

Einen weiteren Beitrag zu Schopenhauers Metaphysik der Sitten versucht de L o r e n z o mit seinem Aufsatz: Schopenhauer, Byron und Leopardi zu geben:

Ausgehend von den vier heiligen Wahrheiten Gotama Buddhas sucht er nachzuweisen, daß Leopardi nur die erste gekannt, Byron auch die zweite richtig gefühlt, jedoch erst Schopenhauer alle vier ganz erfaßt habe. Bedauerlich ist Lorenzos En:gleisung am. Schluß der Arbeit, an welcher Stelle er Buddha als Philosophen über Schopenhauer stellt. Er übersieht, daßß Buddha nicht Philosoph ist und keiner sein will. Gotama fühlt die nämlichen Wahrheiten, die dann Schopenhauer deutet. Sie miteinander zu vergleichen, ist also in diesem Sinne nicht angängig. 
Mit Schopenhauers Metaphysik der Natur befaßt sich außerordentlich glücklich Prof. L u bos ch. Um Schopenhauers Übereinstimmung mit den Naturforschern seiner Zeit-klarzumachen und zugleich seine Abweichungen, die Lubosch sehr richtig als Vertiefungen kennzeichnet, uns vor Augen zu stellen, wird uns das Programm des Anatomen Döllinger vorgeführt, welches nach Lubosch geradezu eine Interpretation der. Naturphilosophie Schopenhauers liefert. Sehr klar und überzeugend führt Lubosch die sich selbst gestellte Aufgabe durch. Bedauerlich bleibt nur eines: Bei Anlaß der Feststellung, daß Schopenhauers Abweichungen lediglich Vertiefungen seien, begibt sich Lubosch gerade der folgenschwersten, klärendsten der Schopenhauerschen Vertiefungen, indem er es verschmäht, Schopenhauers Ideenlehre in ihrer ganzen Tiefe zu beriicksichtigen. Sie gerade aber liefert den Hauptschlüssel, der alle scheinbar trennenden Türen sprengt. Hier gerade liegt die Verknüpfung des Evolutionisten in Schopenhauer mit dem gleickzeitigen Bekenner des wandellosen Dinges an sich. Schopenhauer ist an keinem andern Punkt seiner Lehre so sehr wie gerade hier für die Naturwissenschaftler ,der Lehrer echt wissenschaftlichen Sinnes", wie Lubosch ihn in dem bündigen Schlußsatz seines Aufsatzes nennt, welcher gleichzeitig trefflich den heutigen Stand und die Fehler der Naturforschung kennzeichnet:

. . . . „er ist uns auch ein Lehrer echt wissenschaftlichen Sinnes geworden, indem er uns zeigte, daß man die Ätiologie nicht durch eine philosophische Erklärung ersetzen dürfe, wië es die Naturphilosophen seinerzeit taten; aber ebenso wenig die philosophische Erklärung durch eine vermeinłlich ätiologische. Diese letztere heute leider so verbreitete Methode naturwissenschaftlicher Forschung hätte ihn unter ihre Anhänger schwerlich zu rechnen gehabt." S. 127.

Schopenhauers Metaphysik des Schönen haben Gotthelf und Hiller Aufsätze gewidmet.

Gotthelf schreibt in temperamentvoller Weise über Schopenhauers Verhältnis zur Musik, zur Oper, zu Richard Wagner. Der Aufsatz enthält sehr viel klar und richtig geschautes.

Ganz ausgezeichnet ist z. B. mit knappen Worten der Weg skizziert, der Wagner zur Opernreform führte.

In fesselnder dramatischer Steigerung erreicht der Aufsatz einen Gipfelpunkt in der interessanten Gegenüberstellung zweier Austpriche (S. 36): 
Der Irrtum in dem Kunstgenre der Oper besteht darin, daß ein Mittel des Ausdrucks (die Musik) zum Zweck, der Zweck des Ausdrucks (das Drama) aber zum Mittel gemacht war (Wagner) ... daß «s ein großer Mißgriff und eine arge Verkehrtheit sei, wenn in der Oper der Text sich zur Hauptsache und die Musik zum bloßen Mittel ihress Ausdruckes mache (Schopenhauer).

Diese Antinomie läßt sich jedoch auflösen, wie mir scheint. Wagner sagt mit seinem Satz noch keineswegs, daß für ihn die Musik bloß Mittel, und Schopenhauer anderseits behauptet nicht, daß für ihn die Musik bloß Zweck sei. Was Wagner letzten Endes wollte: Gleichordnung, nicht eigentlich Unterordnung, dem hätte vielleicht. auch Schopenhauer bedingt beigestimmt. Seine abfällige Bemerkung über die "Gütergemeinschaft" von Poesie und Musik in Wagners Opern, die er Hebler gegenüber gemacht haben soll, dürfen wir jedenfalls nicht als feststehenden Beweis ansehen. Was Mockrauer S. 164 über den tadelnswerten Mißbrauch der Nachlaßpublikationen zum Beleg für. angebliche Lehren sagt, kann auch hier seine Anwerdung finden. Ich glaube, daß Schopenhauers Abneigung nicht so sehr gegen die Wagnersche Opernform als solche gerichtet war, sondern gegen die Wagnersche Musik, die wohl seinem zwar sehr sensitiven, aber dennoch gesunden Empfindungsleben widerstrebte.

Hiller bringt in einem stilistisch und wortkünstlerisch reizvollen Aufsatz Beiträge zu Schopenhauers Gedanken über die Architektur.

Er findet Schopenhauers Betrachtungen einseitig, weil sie die Bauwerke nur vom ästhetischen aber nicht zugleich vom Zweckstanddunkt aus würdigen.

Aus. diesem Grunde habe Schopenhaner den Begriff des Raumgefïhls nicht beriicksichtigt, er habe die Ingenieurbauten nicht in den Kreis seiner Betrachtungen geżogen und er habe darum auch in der Gothik den seelischen Inhalt und Zweck außer Acht gelassen.

Dagegen ist zu sagen: Auch unsre modernen Begriffe des Raumgefühls und der Raumwirkung lassen sich restlos durch die Schopenhauersche Doppeltheorie aes Verhältnisses von Stütze und Last einerseits und der Offenbarung des Wesens des Lichis anderseits ausdeuten. Ein Raum löst „erfreuliche“ Gefühle aus, wenn Stütze (Mauern) und Last (Decke) in richtigem Verhältnis zueinander stehen. Er wirkt drückend oder kältend, wenn er zu nieder, resp. zu hoch 
im Verhältnis zu seiner Tiefe ist. Auch gelten die Gesetze vom Auffangen, Hemmen und Zurückwerfen des Lichțes ganz gleich für innen wie für außen und schaffen Vornehmheit oder Intimität, Trauer oder Heiterkeit in àer Raumwirkung.

Daß Schopenhauer aie „Ingenieurbauten" nicht berïcksichtigt habe, stimmt nicht. Siehe Vorl. X, 275, wo er von Eisenkonstruktionen spricht.

Hillers von Schopenhauer abweichende Beurteilung der Gothik beruht auf einer Verwechselung und Verquickung der Ästhetik mit der Ethik. Jedem Menschen erscheint wohl sein Vaterhaus schön. Ob es an sich, den Gesetzen der Ästhetik entsprechend, schön ist, das ist eine andere Frage.

Hiller scheint einer von jenen, die an eine Entwicklung in allem und jedem glauben; bei denen wohl auch der Stein das Fallen ,gelernt" haben muß. Darum ist ihm der Gedanke unerträglich, daß es für die Baukunst aus Steị keine nemnenswerte Entwicklung mehr geben sollte. Und doch ist dem so. Das Verhältnis von Stütze und Last läßt sich nicht ins Unendliche variieren. Deshalb bleiben aber der Entwicklungsmöglichkeiten genug, da immer neue Baumaterialien gefunden werden können. So sagt Schopenhauer selbst in den Vorlesungen X S. 275: „Die Proportionen, welche für steinerne Gebäude und ihre Teile als die besten befunden worden, sind nicht auf das Eisen sofort anwendbar: daher müßte man für die schöne Baukunst aus Eisen andre Säulenordnungen und andre Regeln überhaupt erfinden."

Hier liegt Entwicklungsmöglịchkeit im Ganzen vor. Unabșehbar aber ist die Aufgabe im Einzelnen: Schärfung des Empfịndens für die Gesetze der Schwere und des Lichtes; Erziehung zur Erfassung des großen Baues als eines geschlossenen Ganzen und damit die stete Wiedererweckung von Fähigkeiten, die unserm Geschlecht der Spezialwissenschaften immer mehr abhanden zu kommen drohen.

Neben den rein philosophischen Arbeiten stehen einige philologische, unter denen Mockrauers Bericht über Schopenhauers Erstlingsmanuskripte an der Spitze steht.

Er findet warme Worte des Einfühlens in Schopenhauers Denkund Arbeitsweise, lebhafte Ausdrücke der Bewunderung: des überragenden Geistes. Ausgezeichnet ist S. 141 die Charakterisierung des Schopenhauerschen Willensbegriffs. Leider zeigt sich aber auch 
in dieser Arbeit an mehreren Stellen mangelnde Kenntnis der philosophia prinia; so S. 143, wo die von Mockrauer aufgestellte Hypothese der Entwicklung des Schopenhauerschen Willensbegriffes die Keime der für viele Apostel so verhängnisvollen Verwechselung des erkenntnistheoretischen a priori mit dem ethischen Verneinungsbegriff durchblicken läßt. Desgleichen zeugt die Ausführung S. 144 von einer Verkennung der eigentlichen Basis der Ideenlehre, wie denn auch der Nachweis hierzu S. 154 und 155 sich selbst aufhebt, da schon in der ersten Stelle enthalten ist, was Mockrauer erst in der zweiten als neu zu finden glaubt. Auch das alte Märchen von einer Umwandlung der Schopenhauerschen Philosophie der W I in W II wird hier wieder aufgetischt. Unkenntnis der philosophia prima verrät auch Mockrauers Zustimmung zur Lehre vom transzendentalen Subjekt (S. 160).

Doch ist, abgesehen von diesen Entgleisungen die Abhandlung als eine gute Einführung in die in Bälde erscheinenden Erstlingsmanuskripte anzusehen. Zieht auch durch die ganze Arbeit wie ein Leitmotiv der Gedanke: Über Schopenhauer hinaus; so dürfen wir wohl solche Überhebung dem jugendlichen Enthusiasmus des Verfassers zu gute halten und wir können uns im übrigen der schönen Arbeit, die uns Schopenhauer näher zu bringen sucht, aufrichtig freuen.

Eine rein philologische Arbeit von nicht großem Wert ist Merbachs: Schopenhauer und die theoretischen Grundlagen der Schauspielkunst.

Weit geringer noch an Wert und köstlich in seiner Naivität ist Ladds Aufsatz: Schopenhauer and America, der gerade das Gegenteil von dem beweist, was er darzulegen beabsichtigt: daß nämlich Schopenhauer mit seinem Urteil über die Amerikaner nicht etwa irrt, sondern daß er damit nur allzu recht hat. Daß Amerika mit der Negerfrage nicht fertig wird, zejgt ja gerade, daß drüben eben die geistige Superiorität fehlt, die allein das Dilemma zu lösen imstande wäre.

Zwei Aufsätze mehr persönlicher Natur sind die Samuelsons und Deussens.

Samuels.on versucht einen Wegweiser zum Lesen Schopenhauers für Nichtfachleute zu geben. Es mag zwar scheinen, daß ich mir in Folgendem sellbst widerspreche; ich sage es aber dennoch: Meiner Meinung nach sollten Nichtfachleute nicht mit der philosophia 
prima beginnen. Für sie rate ich folgenden Weg, um mit Schopenhauer bekannt zu werden:

Parerga I von hinten nach vorn; Parerga II von hinten nach vorn; dann W 2, Ergänzungen zu Buch 4, dann zu Buch 3; dann W 1, Buch 4 und 3; dann die beiden Preisschriften; dann W 2, Ergänzungen zu Buch 2; dann W 1, Buch 2, dann über den Willen in der Natur; zuletzt W 1 und 2, Buch 1 und Ergänzung; zum Schluß die Kritik Kants und den Satz vom Grunde. Zwischen N und W 1, Buch 1, würde ich empfehlen, Kants transzendentale Ästhetik, die Methodenlehre, dèn 4. Paralogism und die Prolegomena zu studieren; ev. auch die Kritik der spekulativen Theologie; daneben dann nochmals die ersten Aufsätze in P I. Dieser Weg hat den Vorteil, vom leichtverständlichen zum schwereren fortzuschreiten; wer dann vom Nichtfachmann zum Fachmann werden will, halte sich zum grïndlichen zweiten Durcharbeiten an die von Samuelson angegebene Reihenfolge.

De ussen versucht in seiner Vorrede einen Wegweiser anderer Art. Er geht mit Schopenhauer durch unsere heute so bewegte Zeit Der Aufsatz ist außerordentlich erfreulich und zeigt, wie richtig, wie klar ein Auge sieht, das durch Schopenhauer geschärft ist; es erkennt im Zufälligen das Typische, im Zeitlichen das Zeitlose; im Streit den Frieden.

Wie trefflich formuliert er das Programm der Gesellschaft für diese Zeit des Sturmes: „,erhaltend und versöhnend auf den Gang der Ereignisse einwirken“; wie mutig geißelt er die Torheit einiger Professoren, die ihre ausländischen Ehrungen zurückgesandt; wie klar kennzeichnet er die wahren Urheber des Krieges! - Wie Fanfaren= ruf klingt seine Losung: „So gewiß Schopenhauers Philosophie, wenn auch erst nach Jahrhunderten, alle Strömungen des geistigen Lebens $z u$ beherrschen und $z u$ durchdringen berufen ist, so gewiß haben diejenigen, welche schon heute ihrer unschätzbaren Wohltat teilhaft geworden sind - vergleichbar den Gipfeln der Berge, welche von der aufgehenden Sonne beschienen werden, wenn die Täler noch in Nacht und Dunkel liegen - es als eine heilige Pflicht zu empfinden, das geistige Licht, welches ihnen zuerst vor vielen andern geschenkt worden ist, der durch diesen Krieg in Kunst und Wissenschaft hereinbrechenden Barbarei gegenüber $\mathrm{zu}$ pflegen und $\mathrm{zu}$ wahren." VI/VII. 
Zwei Künstler haben sich diesmal unter die Schar der Wissenschaftler verirrt, - wie cin Gletscherbach, fortreißend, sprudelnd, kraftvoll und rauschend, klingt der Strom ihrer Künstlersprache wohltuend, befreiend, begliickend an unser $\mathrm{Ohr}$ :

Gjellerup und Bahr. - Und es sind echte Künstler; was sie uns schenken, ist Wahrheit. - Vom echten Künstler aber wird die Währheit nicht erarbeitet, sondern erlebt, gefühlt. ..

Woran sich die Wissenschaftler die Zähne ausbeißen: am Problem der Heiligkeit: Gjeller up fühlt's. Er spürt mit der nachtwandlerischen Sicherheit intuitiven Erkennens die Wurzeln aller Blüten in der philosophia prima: „Die positive Seite der Schopenháuerschen Religionsphilosophie ist die zentrale Stelle. Denn sie ist der Angelpunkt, in welchem Schopenhauers Naturdeutung in seine Heilslehre umschwingt."

Seine kurzen Bemerkungen über den kategorischen Imperativ und über das „bessere Bewußtsein“, und seine geniale Gegenüberstellung der Begriffe Schöpfergott und Entfaltergott beweisen, daß dieser Küns.ler weit tiefer das Wesen und den wahren Sinnn der Schopenhauerschen Philosophie erfaßt hat, als die meisten Wissenschaftler im selben Buche. Und wie ein echter Künstler gibt er das fließende Bild der Zeit uns plastisch wieder mit den Worten: „Hier (Deutschland-Österreich) der ethische Idealismus. Dort: im Osten diffuser ikonolatristischer Aberglaube im Banne des absoluten Knutentums, im Westen das gänzlich ideelose Strebertum der dritten Republik. Und endlich - last not least - auf seinem Isolierschemel thronend, der reinhumanitäre Utilitarismus, mit obligater Begleitung jener verflachten anglikanischen Karrikatur des Christentums, gegen welche unser Meister einige seiner heftigsten Philippika gerichtet hat."

Ganz anders als Gjellerup und doch im tiefsten Innern, als Künstler, mit ihm verwandt, schaut Hermann Bahr Schopenhauer an. Er lehnt dessen. Weltanschauung ab, denn er kann nicht auf eine andre als seine eigene Fahne schwören. Und von seinem Künstlerstandpunkt aus hat er darin völlig recht.

Alle ,gefühlte ". Wahrheit ist rein individuell.

Bahr, der geniale. Einfühler und treffsichere Psycholog, fühlt ein Widerstreben gegen alles Aufgebaute, Konstruierte, wissenschaftlich Bewiesene. Er braucht das abstrakte Erkennen nicht, da ihm das intuitive in so seltenem Maße eignet. 
So tritt er denn auch an Schopenhauer nur mit dem Maßstab des intuitiven Erkennens heran; läßt alles liegen, was Anstrengung kostet, nimmt nur an, was unmittelbar und mühelos volles Echo in seinem eigenen Innern findet. Und so liebt.er denn an Schopenhauer am meisten, daß dieser ,sein Inneres hat ganz erscheinen lassen und rein". "Wovon er spricht, er ist immer derselbe und überall ist er ganz."

Beglückend ist es aber für uns Wissenschaftler, hier die Schlußfolgerungen zu ziehen, die Bahr, den Künstler, nicht reizen:

Alle großen Wahrheiten sind zunächst ,gefühlte“. Je restloser sie bewiesen werden können, je weniger Antinomien nach ihrer wissenschaftlichen Festlegung übrigbleiben, desto näher sind sie der Wahrheit gekommen.

Nun ist, wie Bahr intuitiv richtig fühlt, Schopenhauer einer der ganz seltenen Einheitlichen, ganz durchsichtig Klarew. Uñd daraus läßt sich nun der Schluß auf die Lückenlosigkeit, die Echtheit, die Einzigartigkeit der uns durch ihn geschenkten Weltanschauung ziehen:

Weil Schopenhauer wie kein zweiter ,die ganze. Welt zum Stoff nahm, sich darin einheitlich abzubilden, weil er nichts ,berïhrt, das nicht zu seinem Zeichen würde", darum muß - es kann ja nicht anders sein - eine also lückenlos bewiesene Wahrheit auch die richtig gefühlte - die' Währheit - sein.

Und darum sollte als wichtigste Mahnung über jeder Seite eines jeden neuen Schopenhauer-Jahrbuchs geschrieben stehen:

Nicht über ihn hinaus, sondern in ihn hinein. 